\title{
Mixossarcoma esplênico em cão
}

\author{
Splenic Myxosarcoma in a Dog \\ Taís Teixeira Zambarda', Mariana de Jesus de Souza², Jéssica Moreira Cannavon', \\ Clarisse Felten', Saulo Petinatti Pavarini ${ }^{3}$ \& Álan Gomes Pöppl ${ }^{4}$
}

\begin{abstract}
Background: Myxosarcomas (MXS) are malignant mesenchymal neoplasms originated from mainly mucin producing fibroblasts than collagen. Despite malignant neoplasms, MXS is believed to have low metastase generation ability. Myxosarcomas reports in dogs are widespread, usually involving trunk and limbs subcutaneous tissue. The aim of this report is to describe clinical, pathological, and immunohistochemistry aspects of a rare case of splenic myxosarcoma in a dog.

Case: A 10-year-old, intact male Basset Hound, weiging $25 \mathrm{~kg}$, showing signals such apathy, dark soft-stooled stools, progressive thinning, and increasing abdominal enlargement, perceived three months ago was brought to clinical consultation. Physical examination showed extremely pale oral and ocular mucosas, in addition to mild dehydration $(6 \%), 35.4^{\circ} \mathrm{C}$ rectal temperature, and a very bulging abdomen, with no fluids or gases, evidence at abdominal percussion. A complete blood count (CBC) revealed neutrophilic leukocytosis and anemia. Patient's abdominal cavity ultrasound examination showed an heterogeneous mass, occupying large part of cavity, and apparently from spleen origin. A spleenic multilobulated mass measuring $25 \times 12 \times 20 \mathrm{~cm}$ was obtained after exploratory laparotomy. The mass showed predominantly soft consistency with some firm areas, and no visible mesentery or other abdominal organs adhesions. Mass and spleen histopathological examination were routinely processed for histological analysis with haematoxylin and eosin and alcian blue staining. In addition, fragments of the neoplasm were submitted to immunohistochemistry (IHC) which confirmed myxosarcoma diagnosis. The owner returned to hospital two months after, complaining for apathy, prostration, anorexia, and abdominal pain. A new ultrasound examination were suggestive of liver and kidneys neoplasms. The tutor opted for patient's euthanasia. However, dog's necropsy was not authorized.

Discussion: Myxosarcomas are malignant tumors considered rare in small animals, affecting mainly middle age or advanced age patients. Corroborating with the present case, the main clinical signs identified in cases of splenic neoplasias, in general, are abdominal distention and algia, emaciation, prostration, anemia, hypothermia, and inappetence. Regarding neutrophilic leukocytosis and anemia in $\mathrm{CBC}$, those alterations are atributable to paraneoplastic syndromes commonly observed in small animals. The diagnosis of myxosarcoma was based on the pathological and IHC findings. The mesenchymal origin of the neoplastic cells was confirmed by the expression of vimentin and by the absence of cytokeratin labeling in the immunohistochemical examination. It was possible to confirm the diagnosis due to the presence of large amount of mucopolysaccharides between the neoplastic cells, demonstrated through Alcian Blue staining. Although rare, there have been reports of metastasis from cardiac myxosarcoma in humans and splenic myxosarcomas in dogs. The patient's resubmission in two months with a new nonspecific clinical picture and documentation of hepatic and renal masses supports the possibility of agressive metastatic disease in the present case, despite the non-authorization of the autopsy examination. It was concluded by the present case report that MXS, although uncommon in small animals, should be included as a differential diagnosis in cases of abdominal neoplasias. The histopathological analysis and the immunohistochemical examination of neoplasms are important and should be considered in order to establish diagnosis and prognosis in dogs. Despite not confirmed by post-morten examination, agressive metastic disease seems likely in the present report, and emphasize MXS'malignat nature.
\end{abstract}

Keywords: immunohistochemistry, mesenchimal neoplasm, soft tissue sarcoma.

Descritores: imuno-histoquímica, neoplasia mesenquimal, sarcoma de tecidos moles.

1Programa de Residência em Área Profissional da Saúde em Saúde Animal e Coletiva, Hospital de Clínicas Veterinárias (HCV), Universidade Federal do Rio Grande do Sul (UFRGS), Porto Alegre, RS, Brazil. ²Programa de Pós-graduação em Ciências Veterinárias (PPGCV), Faculdade de Veterinária (FaVet), UFRGS, Porto Alegre. ${ }^{3}$ Setor de Patologia Veterinária (SPV), FaVet, UFRGS, Porto Alegre. ${ }^{4}$ Departamento de Medicina Animal, FaVet, UFRGS, Porto Alegre. CORRESPONDENCE: T.T. Zambarda [taiszambarda.vet@gmail.com - Tel.: +55 (51) 98231-4282]. Faculdade de Veterinária - UFRGS. Av. Bento Gonçalves 9090, Bairro Agronomia. CEP 91540-000 Porto Alegre, RS, Brazil. 


\section{INTRODUÇÃO}

Mixossarcoma (MXS) é um neoplasma mesenquimal maligno, pouco frequente em cães e gatos, sendo classificado como sarcoma de tecidos moles [6]. Originam-se de fibroblastos, células estas que passam a produzir maior quantidade de mucina ao invés de colágeno [4]. Em cães, este tipo de neoplasia tem sido associado à radiação, corpos estranhos, implantes ortopédicos, traumas e parasitismo por Spirocerca lupi. Apesar de neoplasmas malignos, acredita-se que os MXS tenham baixa capacidade de gerar metástases [6]. São esparços os relatos de MXS em cães, geralmente acometendo tecido subcutâneo de tronco e membros [4]. Contudo, acometimento do pavilhão auricular, vesícula urinária, olhos, coração, entre outros locais, já foram descritos $[3,6,8,10]$. Este relato tem o objetivo de descrever os aspectos clínicos, patológicos e imunohistoquímicos de um raro caso de mixossarcoma esplênico em um cão.

\section{CASO}

Um canino macho inteiro, Basset Hound, de 10 anos de idade, pesando $25 \mathrm{~kg}$, foi atendido com histórico de apatia, fezes escuras e de consistência amolecida, e emagrecimento progressivo associado a abdominomegalia crescente, com evolução há três meses. Ao exame físico o paciente apresentou mucosas orais e oculares pálidas, desidratação estimada em $6 \%$, temperatura retal $35,4^{\circ} \mathrm{C}$, e abaulamento abdominal sem evidência de líquido ou gases à percussão abdominal. Exames hematológicos e bioquímicos foram solicitados e demonstraram hematócrito de $20 \%$, acompanhado de anemia normocítica-normocrômica, e leucocitose (64.700 cél/ $\mu \mathrm{L})$ neutrofilíca $(58.230 / \mu \mathrm{L})$ [9]. As concentrações de trombócitos $(231.000 / \mu \mathrm{L})$, proteína plasmática total (80 g/L), creatinina $(0,7 \mathrm{mg} / \mathrm{dL})$, uréia $(43 \mathrm{mg} / \mathrm{dL})$ e a atividade sérica da alanina aminotransferase (ALT) (29 UI/L) encontravam-se dentro dos valores de referência para a espécie [5]. Foi realizada ultrassonografia abdominal do paciente, que revelou a presença de uma massa heterogênia, ocupando grande parte da cavidade, aparentemente originada do baço, sem evidência de líquido livre e de alterações dignas de nota nos demais órgãos da cavidade. Exame radiográfico do tórax revelou ausência de lesões metastáticas.

Após prova de compatibilidade, o paciente recebeu transfusão sanguínea, na dose de $20 \mathrm{~mL} / \mathrm{Kg}$, e posteriormente foi encaminhado para laparotomia explo- ratória. Uma massa medindo 25 x 12 x $20 \mathrm{~cm}$, oriunda do baço, multilobulada e de consistência predominantemente macia com algumas áreas firmes, sem aderências visíveis ao mesentério e demais órgãos abdominais, foi visualizada durante a cirurgia. Foi realizada esplenectomia e a massa juntamente com o baço foi enviada para exame histopatológico. Ao corte a massa apresentava áreas gelatinosas de coloração cinza entremeadas por áreas amareladas levemente firmes (Figura 1A). A amostra foi processada rotineiramente para análise histológica com colorações pelas técnicas de Hematoxilina e Eosina, e Azul de Alcian. Adicionalmente fragmentos do neoplasma foram submetidos a exame de imuno-histoquímica (IHQ) utilizando os anticorpos primários: vimentina $^{1}$, citoqueratina $\mathrm{AE} 1 / \mathrm{AE}^{2}{ }^{2}$ e S-100². A visualização da imunomarcação foi feita pelo tratamento das lâminas com solução de 3`3-diaminobenzidina (DAB) ${ }^{2}$. Histologicamente observou-se proliferação neoplásica maligna de células mesenquimais arranjadas em feixes frouxos (Figura 1B) entremeados por abundante material mixoide (evidenciada pela coraloração de azul de alcian, Figura 1C). As células eram fusiformes, com citoplasma eosinofílico alongado, núcleos achatados e nucléolos evidentes e exibiam acentuada anisocitose e anisocariose. Observou-se em média três figuras de mitose por campo de maior aumento (400x) além de extensas áreas de necrose intratumoral. No exame de IHQ, as células neoplásicas apresentram acentuada marcação citoplasmática para vimentina (Figura 1D) e foram negativas para citoqueratina e S-100. Os achados do exame indicaram tratar-se de um MXS esplênico.

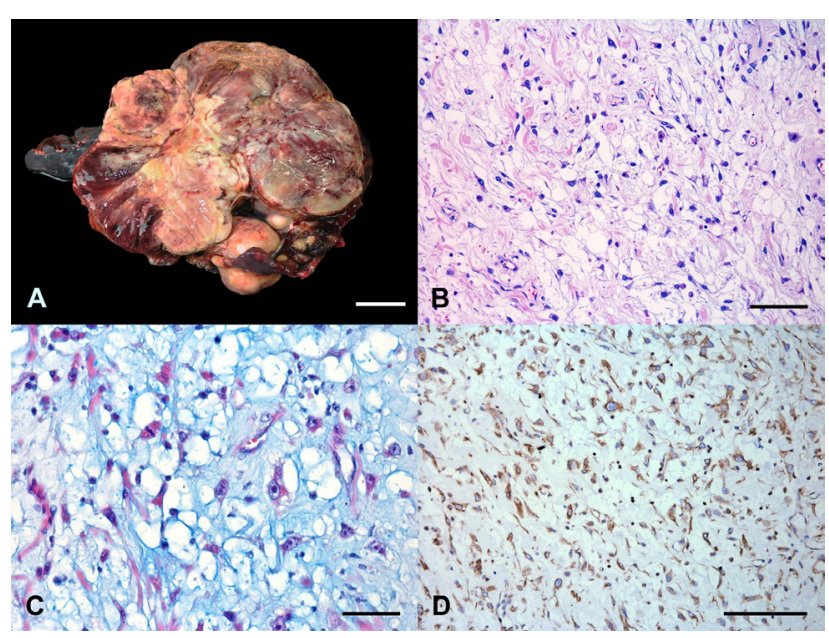

Figura 1. Mixossarcoma esplênico em um canino. A- Baço com uma massa multilobulada medindo $25 \times 12$ × $20 \mathrm{~cm}$ de consistência macia, com áreas gelatinosas de coloração cinza entremeadas por áreas amareladas levemente firmes [Barra $=4 \mathrm{~cm}]$. B- Células neoplásicas fusiformes, arranjadas em feixes frouxos entremeados por abundante material mixoide [HE, Barra $=160 \mu \mathrm{m}]$. CEvidenciação de material mixoide entre as células neoplásicas pela coloração de Azul de Alcian [Barra= $60 \mu \mathrm{m}$ ]. D- Imunomarcação acentuada para vimentina no citoplasma das células neoplásicas. Cromógeno DAB [Barra= $350 \mu \mathrm{m}]$. 
Ao longo dos cinco dias de pós-operatório o paciente foi mantido em fluidoterapia a base de Ringer Lactato $(50 \mathrm{~mL} / \mathrm{kg} / \mathrm{dia}, \mathrm{IV})$, metadona $(0,3 \mathrm{mg} / \mathrm{kg}$, QID, SC, por 2 dias) e subsequentemente cloridrato de tramadol (4 mg/kg, TID, SC, por 3 dias), cefalotina (20 $\mathrm{mg} / \mathrm{kg}$, TID, IV), enrofloxacino (5 mg/kg, BID, IV) e metronidazol (20 mg/kg, BID, IV). Em $24 \mathrm{~h}$ da cirurgia documentou-se melhora parcial da leucocitose pelo cão (41.000/ $\mu \mathrm{L})$ e aumento do hematócrito (28\%). Devido a relevante melhora do quadro clínico geral, além de exames laboratoriais indicando hematócrito de $36 \%$, 27.000/ $\mu \mathrm{L}$ leucócitos totais, sendo $24.030 / \mu \mathrm{L}$ neutrófilos segmentados, e $230.000 / \mu \mathrm{L}$ plaquetas, no sexto dia pós-opertaório o paciente teve alta. Prescreveu-se então cloridrato de tramadol (3 mg/kg, TID, VO, 5 dias), amoxicilina com clavulanato de potássio $(20 \mathrm{mg} / \mathrm{kg}$, BID, VO, 10 dias), omeprazol (1 mg/kg, SID, VO, 10 dias). Após sete dias o paciente foi reavaliado apresentando-se estável, ativo, e com parâmetros clínicos inalterados. Em dois meses o cão retornou para atendimento, sendo relatada queixa de apatia, prostração e anorexia. Ao exame clinico constatou-se algia abdominal e presença de estrutura arredondada à palpação em região de silhueta hepática. Exames de imagem evidenciaram fígado com dimensões aumentadas, com múltiplos nódulos distribuídos pelo parênquima, o maior medindo $5 \mathrm{~cm}$ x $2,7 \mathrm{~cm}$, e múltiplas áreas arredondadas hiperecogênicas em região cortical de ambos os rins, a maior com cerca de $1 \mathrm{~cm}$. Diante do quadro clínico e dos resultados dos exames de imagem, os quais foram sugestivos de neoplasias em fígado e rins, os tutores optaram pela eutanásia do paciente e não autorizaram exame de necropsia.

\section{DISCUSSÃO}

MXS são tumores malignos considerados raros em pequenos animais e como no presente caso, afetam principalmente animais de meia idade ou idade avançada [6]. No entanto, sua apresentação esplênica não é usual. Um estudo analisou 173 baços de cães esplencetomizados, e somente um animal apresentou MXS. Os sinais clínicos identificados em casos de neoplasias esplênicas, em geral, são semelhantes aos apresentados no presente caso. Tratam-se principalmente de distensão e algia abdominal, emagrecimento, prostração, anemia, hipotermia, e inapetência [1]. Leucocitose neutrofílica e anemia são síndromes paraneoplásicas comumente observadas em pequenos animais e justificam as alterações laboratoriais documentadas neste paciente. No entanto, estas manifestações clínicas e laboratoriais são demasiado inespecíficas, sendo o diagnóstico final inesperado no presente caso. Além da retirada do tumor, casos de neoplasias intra-abdominais podem necessitar de transfusões sanguíneas conforme a gravidade da anemia, como observado no presente relato [2].

O diagnóstico de MXS no canino desse relato baseou-se nos achados patológicos e imuno-histoquímicos. A origem mesenquimal das células neoplásicas foi comprovada através da expressão de vimentina e pela ausência de marcação para citoqueratina no exame de IHQ. O principal diagnóstico diferencial nesse caso é o fibrossarcoma esplênico, que também seria positivo na IHQ para vimentina. Todavia, a presença de grande quantidade de mucopolissacarídeos entre as células neoplásicas, demonstrada através da coloração de azul de alcian, corrobora na confirmação do diagnóstico de MXS no presente caso [4].

Apesar de consideradas raras [4,6], há relatos de metástases oriundas de MXS cardíaco em humanos, e de MXS esplênico em cães [7,11]. A reapresentação do paciente em dois meses com novo quadro clínico inespecífico associado a documentação de massas hepáticas e renais ao ultrassom suporta a possibilidade de doença metastática agressiva no presente caso, apesar da não autorização do exame de necropsia. $\mathrm{O}$ prognóstico de pacientes com MXS em geral é pobre, podendo-se aplicar o índice mitótico do tumor para esta estimativa. Além disso, este caso corrobora com estudos que relatam grande ocorrência de eutanásia de pacientes com MXS, devido ao prognóstico e consequências advindas da neoplasia [11].

Conclui-se com o presente relato de casos que MXS, apesar de incomuns em pequenos animais, devem ser incluídos como diagnóstico diferencial em casos de neoplasias abdominais. A análise histopatológica e o exame de IHQ de neoplasias são importantes e devem ser considerados para que seja estabelecido diagnóstico e prognóstico em cães. Além disso, apesar da não realização de exame de necropsia, a ocorrência de doença metastática agressiva é bastante provável no presente caso, especialmente em decorrência da localização esplênica da neoplasia primária.

\section{MANUFACTURERS}

1Zymed-Advanced Medical Products Ltda. Santiago, Chile. ${ }^{2}$ Dako North America Inc. Carpinteria, CA, USA.

Declaration of interest. The authors report no conflicts of interest. The authors alone are responsible for the content and writing of the paper. 


\section{REFERENCES}

1 Bandinelli M.B., Pavarini S.P., Oliveira E.C., Gomes D.C., Cruz C.E.F. \& Driemeier D. 2011. Estudo retrospectivo de lesões em baços de cães esplenectomizados: 179 casos. Pesquisa Veterinária Brasileira. 31(8): 697-701.

2 Bergman P.J. 2013. Paraneoplastic Syndromes. In: Withrow S.J. \& MacEwen E.G. (Eds). Small Animal Clinical Oncology. 5th edn. Saint Louis: Elsevier Saunders, pp.83-97.

3 Braz P.H., Martins A.M.Q. \& Souza A.I. 2015. Dificuldades no diagnóstico do mixossarcoma de vesícula urinária em cão. Acta Veterinaria Brasilica. 9(2): 171-175.

4 Goldschimdt M.H. \& Hendrick M.J. 2002. Tumors of the skin and soft tissues. In: Meuten D.J. (Ed). Tumors in Domestic Animals. 4th edn. Ames: Iowa State Press, pp.84-117.

5 González F.H.D. \& Silva S.C. 2006. Introdução à bioquímica clínica veterinária. 2.ed. Porto Alegre: Editora UFRGS, 364 p. 6 Liptak J.M. \& Forrest L.J. 2013. Soft tissue sarcomas. In: Withrow S.J. \& MacEwen E.G. (Eds). Small Animal Clinical Oncology. 5th edn. Saint Louis: Elsevier Saunders, pp.356-380.

7 Kimura M., Kikuchi C., Takahashi Y., Kanazawa H. \& Nakazawa S. 2014. Cardiac myxosarcoma with thoracic spinal metastasis. General Thoracic and Cardiovascular Surgery. 62(7): 441-443.

8 Richter M., Stankeova S., Hauser B., Scharf G. \& Spiess B.M. 2003. Myxosarcoma in the eye and brain in a dog. Veterinary Ophthalmology. 6 (3): 183-189.

9 Rizzi T.E., Meinkoth J.H. \& Clinkenbeard K.D. 2010. Normal hematology of the dog. In: Weiss D.J. \& Wardrop K.J. (Eds). Schalm's Veterinary Hematology. 6th edn. Ames: Blackwell Publishing, pp.799-810.

10 Ruberti B., Rocha D.C.C., Fraiz F.V., Araújo C.M.T.D., CASTRO J.L.C. \& MIARA L.C. 2015. Mixossarcoma em pavilhão auricular de cão: relato de caso. In: $42^{\circ}$ Congresso Brasileiro de Medicina Veterinária e $1^{\circ}$ Congresso Sul-Brasileiro da ANCLIVEPA (Curitiba-PR). pp.1555-1559.

11 Spangler W.L., Culbertsoann M.R. \& Kass D.P.H. 1994. Primary mesenchymal (nonangiomatous/nonlymphomatous) neoplasms occurring in the canine spleen: anatomic classification, immunohistochemistry, and mitotic activity correlated with patient survival. Veterinary Pathology. 31: 37-47. 